\title{
NANO MICRO
}
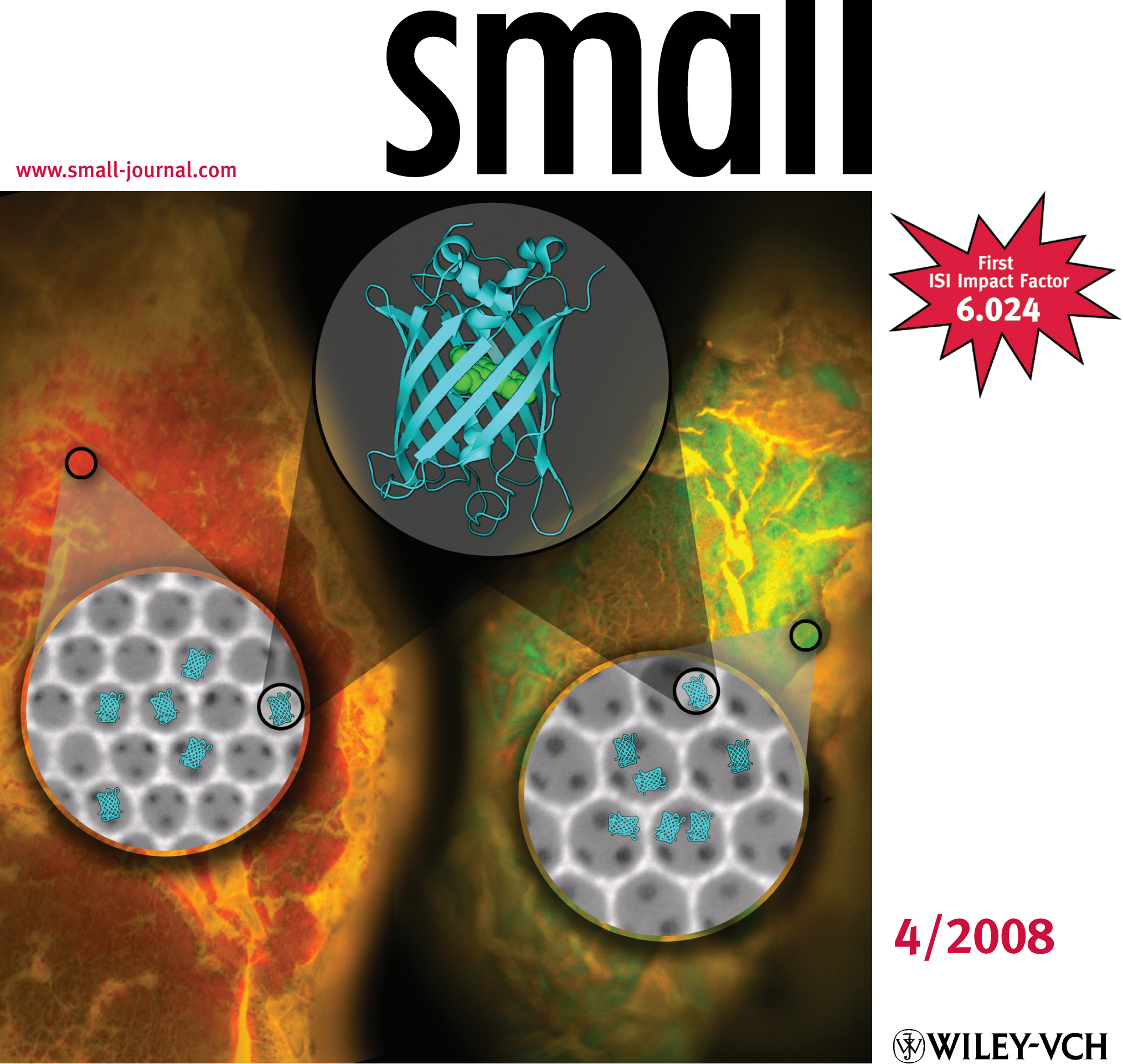

$4 / 2008$

Color Control of Natural Fluorescent Proteins by Photonic Crystals V. Subramaniam, W. L. Vos et al. Nanowires Enabling Signal-Enhanced Nanoscale Raman Spectroscopy M. Becker et al. 
DOI: $10.1002 / \mathrm{smll} .200701160$

\title{
Color Control of Natural Fluorescent Proteins by Photonic Crystals
}

\author{
Christian Blum, Allard P. Mosk, Ivan S. Nikolaev, Vinod Subramaniam, ${ }^{*}$ and \\ Willem L. Vos*
}

\begin{abstract}
The emission of fluorescent proteins inside photonic crystals is studied. It is demonstrated that the apparent emission color of the fluorescent protein can be controlled externally by the photonic crystal. With increasing crystal lattice parameter, the appearance of the proteins turns from orange to red, and suddenly to green. The dramatic color changes agree with the theoretically expected redistribution of light escaping around the stop band of the photonic crystal. Our experiments show the potential of combining biological systems with nanophotonics. This "biophotonic engineering" may be extended to control emission rates and complex Förster energy-transfer systems obtained by protein engineering.
\end{abstract}

\author{
Keywords: \\ - biophotonic engineering \\ - emission control \\ - fluorescent proteins \\ - nanomaterials \\ - photonic crystals
}

\section{Introduction}

The discovery of genetically encodable fluorescent proteins has revolutionized cell biology by enabling the direct visualization of the distribution of macromolecules and their mutual interactions in living cells. ${ }^{[1-3]}$ Protein engineering has been used to tune the color of fluorescent protein emission, thus resulting in a palette of fluorescent proteins emitting from blue to red. ${ }^{[-7]}$ These proteins should be even more useful for photobiological research if their emission properties could be

[*] Dr. C. Blum, Prof. V. Subramaniam

Biophysical Engineering Group

Faculty of Science and Technology and MESA ${ }^{+}$Institute for Nanotechnology

University of Twente

7500 AE Enschede (The Netherlands)

Fax: (+31) 53-489-1105

E-mail: v.subramaniam@utwente.nl

Dr. A. P. Mosk, Prof. W. L. Vos

Complex Photonic Systems (COPS)

Faculty of Science and Technology

and $\mathrm{MESA}^{+}$Institute for Nanotechnology

University of Twente

7500 AE Enschede (The Netherlands)

Fax: (+31) 53-489-5394

E-mail: w.l.vos@utwente.nl

Dr. I. S. Nikolaev, Prof. W. L. Vos

Centre for Nanophotonics

FOM Institute for Atomic and Molecular Physics AMOLF

1098 SJ Amsterdam (The Netherlands) controlled externally. The progress achieved in harnessing light emission by photonic nanostructures ${ }^{[8-11]}$ suggests an elegant control parameter. While intricate natural photonic nanostructures have been identified, ${ }^{[12-14]}$ to date no natural emitters have been discovered whose emission properties are controlled by photonic materials, nor have these systems been realized artificially. Herein, we present the first study of the emission of fluorescent proteins inside photonic crystals. With increasing crystal lattice parameter, the fluorescence of the reef coral fluorescent protein DsRed2 turns from orange to red, and suddenly to green. The dramatic color changes are due to a photonic stop band that shifts through the protein's broad emission spectrum. ${ }^{[15]}$ Our approach enables the combination of the possibilities of protein engineering with the expanding toolbox of nanophotonics.

The unique structure of visible fluorescent proteins and the ability to engineer their photophysical properties has the potential to open new horizons at the interface between biology and the nanosciences. All known fluorescent proteins of this class share the same basic structure of interleaved beta sheets capped by helices and loops to form a cylinder with a diameter of $2.4 \mathrm{~nm}$ and a height of $4.2 \mathrm{~nm}$, as in the first discovered green fluorescent protein. ${ }^{[16]}$ The fluorescent chromophore forms in the center of the protein in an autocatalytic process after transcription and folding of the protein. The encapsulation of the chromophore within a cylindrical protein scaffold (Figure1, left) shields the chromophore from chemical interaction with the protein environment. Thus, collisional quenching and electron 


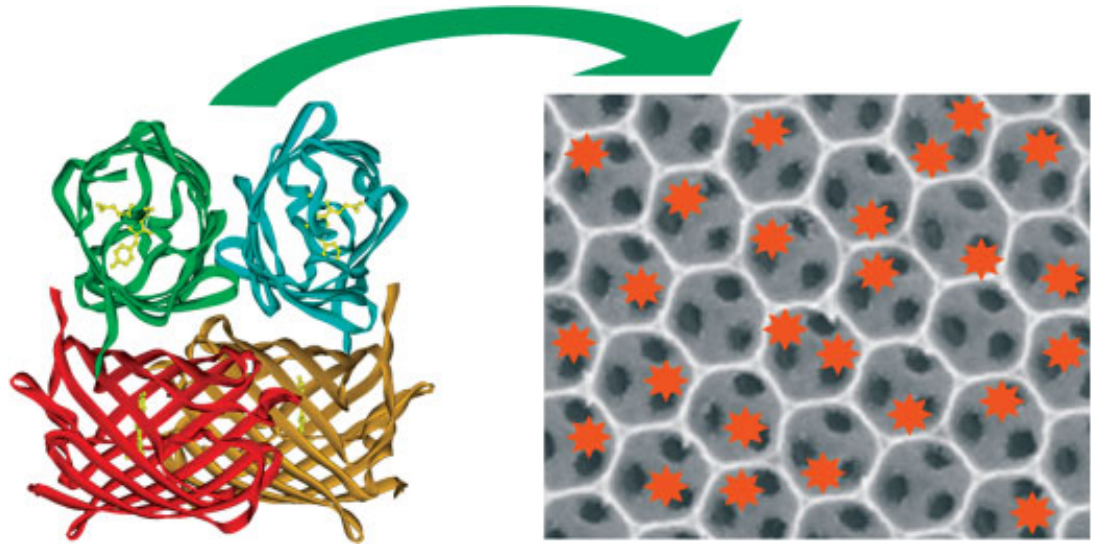

Figure 1. Fluorescent proteins as internal light source in a photonic crystal. Left: Structure of tetrameric red fluorescent protein DsRed2. Each of the four cylindrical monomers forming the tetramer contains one chromophore. The fluorescing chromophore is encapsulated within the cylinder formed by the protein backbone, and is thus shielded from the protein environment. DsRed2 was infiltrated into titania inverse opals. Right: Scanning electron microscopy image of the (111) face of a three-dimensional (3D) titania inverse opal with lattice parameter $a=460 \mathrm{~nm}$. The orange stars symbolize infiltrated fluorescent protein. The small black holes in each air sphere are windows connecting to the next layer of air spheres below. This ensures a 3D connectivity, which is favorable to infiltrate solutions of fluorescent proteins. The titania backbone material is biocompatible.

transfer to the conduction band of the nanostructure are prevented, and the source remains active in environments where other emitters are quenched.

Photonic crystals are currently the subject of intensive theoretical and experimental research as vehicles to manipulate the interaction between light and matter. Such synthetic materials are periodic dielectric composites with spacings comparable to the wavelength of light (Figure 1, right). All photonic crystals control light propagation in particular crystallographic directions by means of Bragg interference, which results in wavelength ranges - called stop bands - for which light is reflected. The appearance of stop bands modifies the emission spectra of embedded light sources and gives rise to directional enhancements or attenuations, in analogy to a lighthouse effect. ${ }^{[17]}$ Photonic crystals also control the density of optical states, which determines the lifetime of excited light sources. In the extreme case of a photonic bandgap where a range of wavelengths is forbidden from propagation in all directions simultaneously, spontaneous emission is completely inhibited, which corresponds to infinite lifetimes. Finally, at the edges of photonic gaps, light propagates with a reduced group velocity, which can stimulate emission ${ }^{[18]}$ and has been reported to enhance the absorption of light in dye-sensitized photovoltaic cells, ${ }^{[19,20]}$ which is relevant to (artificial) photosynthesis. It is thus clear that photonic crystals have interesting prospects to control photobiology.

\section{Results and Discussion}

To explore the interactions between biological macromolecules and artificial photonic nanostructures, we studied the emission color and emission spectra of fluorescent proteins as a function of the lattice parameter of the photonic crystals. We used the DsRed2 variant (Figure 1, left) of the fluorescent protein DsRed isolated from the Discosoma reef coral. ${ }^{[7]}$
Proteins from the DsRed group form tetramers in which four cylindrical monomers form a dimer of dimers arranged in two layers. ${ }^{[21]}$ We infiltrated DsRed2 into titania inverse opals, which are strongly photonic 3D crystals of air spheres in titanium dioxide (Figure 1).

\subsection{Reflectivity and Emission Imaging}

The top row of Figure 2 shows true color images of inverse opals with lattice parameters increasing from left to right. The colorful appearance for each lattice parameter is iridescence, that is, a reflection of the first-order stop bands caused by Bragg diffraction on the (111) crystal planes. ${ }^{[15]}$ With increasing lattice parameter, the crystal appearance changes from blue via green to orange and red, as the stop band shifts from short to longer wavelengths. The middle row of Figure 2 shows true color emission images of fluorescent proteins infiltrated in the same inverse opals. For a small lattice parameter $a=355 \mathrm{~nm}$, the emission reveals a yellow-orange color. This case defines a reference, where the emission spectrum of the proteins is unaffected by the photonic gaps. In this reference crystal, the gaps are at a shorter wavelength $(\approx 485 \mathrm{~nm})$ than the emission of the proteins. For the $a=390 \mathrm{~nm}$ crystal, the protein shows a redder emission for spatial regions that reveal a green iridescence. Conversely, the spatial regions where little or no iridescence occurs emit a yellow-orange color, similar to the reference. The $a=400 \mathrm{~nm}$ crystal also reveals clear red emission from the spatial regions where bright green iridescence occurs. In the $a=445 \mathrm{~nm}$ crystal, the protein emission color has strikingly changed to green, congruent with regions where an orange iridescence is observed. At the left of the image, a reddish emission patch occurs that corresponds to a green iridescence, most likely due to an oblique orientation of the crystal planes in this region. Finally, the $a=460 \mathrm{~nm}$ crystal reveals a bright green emission from the same region as the red iridescence, while noniridescent regions (where crystalline disorder occurs $^{[22]}$ ) again display yellow-orange emission as in the reference. The dramatic changes of the emission colors of the fluorescent protein are controlled by the lattice parameter of the photonic crystals.

\subsection{Emission Spectra}

The bottom row of Figure 2 shows emission spectra from DsRed 2 infiltrated in the corresponding photonic crystals. The emission spectrum from the reference crystal $(a=355 \mathrm{~nm})$ shows that the peak emission wavelength of the protein in the inverse opal is hardly different from that of isolated protein in solution $\left(583 \mathrm{~nm}^{[23]}\right)$. The emission spectrum in the crystal has a full width at half maximum of $85 \mathrm{~nm}$, compared to $50 \mathrm{~nm}$ in solution. We attribute this slight broadening to the loss of 

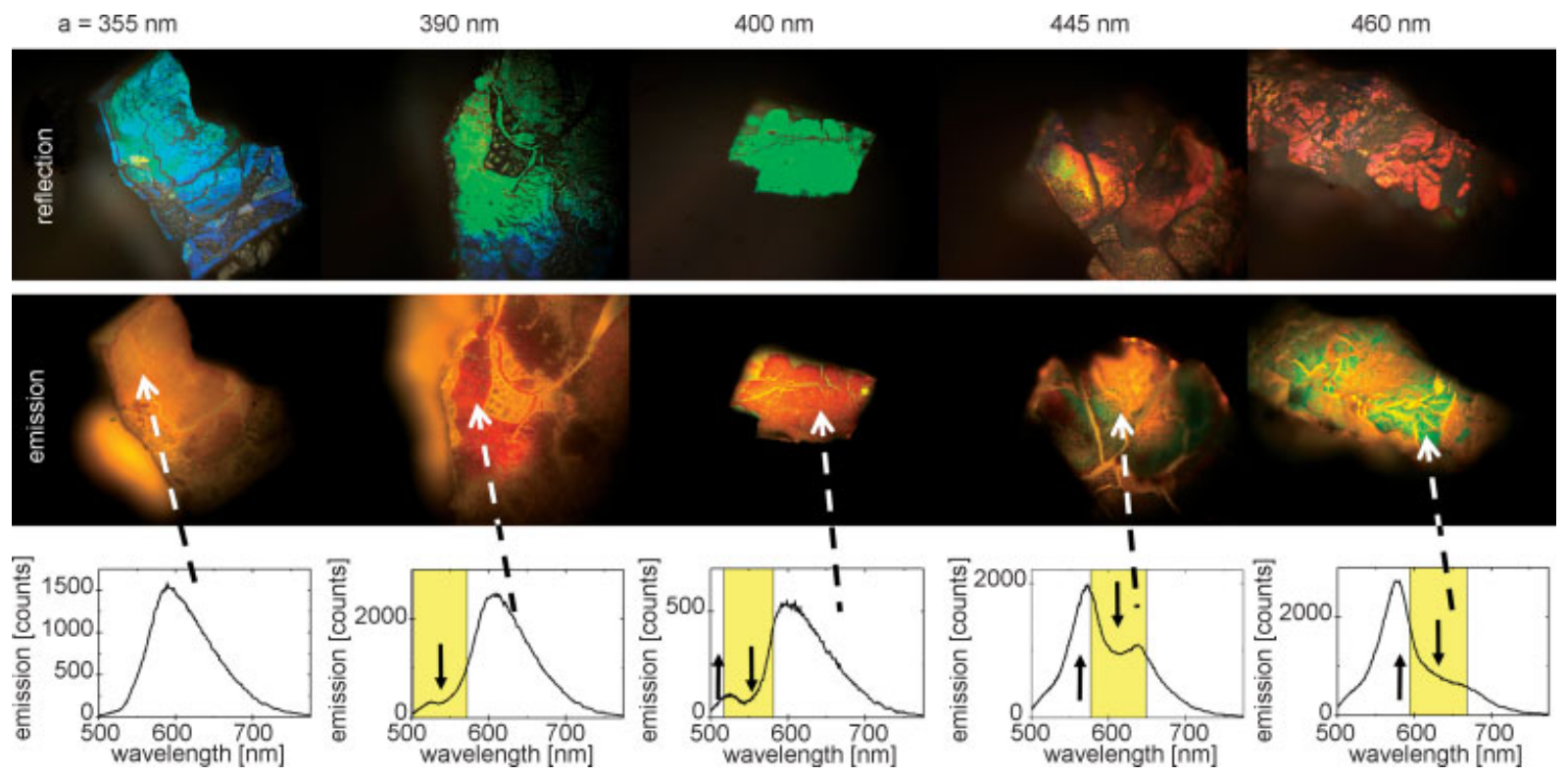

Figure 2. Color appearance of fluorescent protein DsRed2 inside photonic crystals. Top row: Reflection microscopy of titania inverse opal photonic crystals with increasing lattice parameters, as indicated above. Image dimensions are $550 \times 725 \mu \mathrm{m}$. With increasing lattice parameter, the color of the crystals changes from blue via green to orange and red, in qualitative agreement with Bragg's law. Middle row: Fluorescence microscopy of DsRed2 embedded in the same titania inverse opals as the top row. The emission appearance dramatically changes from yellow-orange at $a=355 \mathrm{~nm}$ to red at $a=390$ and $400 \mathrm{~nm}$, to green at $a=445$ and $460 \mathrm{~nm}$. Bottom row: Emission spectra of fluorescent protein DsRed 2 embedded in the same titania inverse opals as above. Bars indicate the photonic stop bands. Solid arrows indicate spectral areas where the emission is enhanced (up) and attenuated (down). Dashed arrows point to the locations (in the fluorescence images) where the spectra were acquired.

water embedded within the protein and possibly to the presence of titania in the close vicinity of the chromophore. As a result, the visible emission from DsRed2 adsorbed and dried on the titania surface appears more yellow-orange than DsRed 2 in solution. The detected emission is intense and commensurate with expectations based on the protein concentration. This finding confirms that the DsRed2 protein barrel protecting the chromophore is still intact even when adsorbed on the inner surface of the inverse opals, since it is known that disturbing the protein cylinder leads to total quenching of fluorescent protein emission. ${ }^{[24]}$ Under identical experimental conditions, undoped crystals reveal a faint emission, which agrees well with the anatase $\mathrm{TiO}_{2}$ luminescence. ${ }^{[25]}$ We find that this contribution of the backbone to the overall detected emission of protein-doped crystals is minor. Therefore, we conclude that the emission spectra represent intact fluorescent protein in photonic crystals.

The emission spectrum for the $a=390 \mathrm{~nm}$ crystal in Figure 2 reveals a trough near $537 \mathrm{~nm}$, which is the result of a wide photonic stop band. ${ }^{[17]}$ Such a broad attenuation of the intensity at the blue side of the emission spectrum results in the red emission color that is indeed observed. The spectrum for the $a=400 \mathrm{~nm}$ crystal reveals an even more pronounced stop band at a wavelength of about $548 \mathrm{~nm}$, closer to the emission peak, and hence the emission color is even more red. For the $a=445 \mathrm{~nm}$ crystal, the spectrum reveals a clear stop band at a much longer wavelength of $612 \mathrm{~nm}$. The red part of the emission spectrum is blocked over a wide range while the green part of the spectrum is enhanced. As a result the emission appears deep green. The green emission color is brighter for the $a=460 \mathrm{~nm}$ crystal, where the stop band has shifted to $630 \mathrm{~nm}$, and the enhancement is more pronounced. These results confirm that the systematic shift of the photonic stop band through the emission spectrum explains the dramatic color changes of the fluorescent protein. The phenomena shown in Figure 2 represent a purely nanophotonic control of the coloration of natural light sources.

\subsection{Quantification of the Modified Emission}

To quantify the effects of the photonic crystals on the emission spectrum of fluorescent proteins, we take the ratio of the emission spectra at selected lattice parameters to the spectrum recorded from the reference sample. We thus obtain a relative intensity spectrum that clearly highlights any attenuation or enhancement of emission relative to the photonically undisturbed spectrum (Figure 3). The data show strong attenuation of $80 \%$ in the photonic stop band as well as a $50 \%$ enhanced emission on the blue side of the stop band, in excellent agreement with the model curve discussed below. The simultaneous observation of attenuation and enhancement means that the effect of the photonic crystals is not a simple filter action; it is the appearance of both attenuation and enhancement within the same spectrum that acts as a photonic contrast enhancement and leads to the remarkably strong emission color changes in Figure 2.

The observed attenuation and enhancement of the DsRed2 emission spectrum (Figure 3) can be quantitatively interpreted with a model for the photon escape probability, which combines diffuse light transport and photonic band structures. ${ }^{[26]}$ The strong attenuation of the spectrum results from Bragg diffraction of light of appropriate wavelength in 


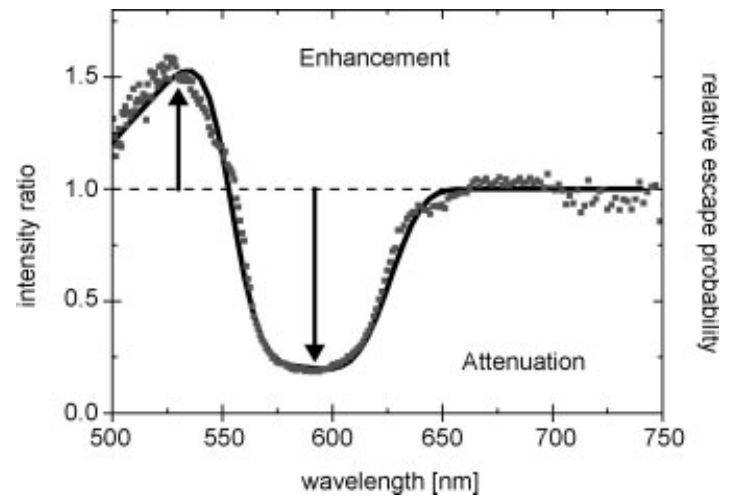

Figure 3. Photonically enhanced and attenuated emission of fluorescent protein DsRed2. Emission spectrum of fluorescent protein in a photonic crystal $(a=430 \mathrm{~nm})$ divided by the emission from a reference crystal. The angle of observation was $0^{\circ}$ relative to the (111) face. An attenuation of $80 \%$ (downward arrow) is apparent in the photonic stop band between 555 and $625 \mathrm{~nm}$. An enhancement of 50\% (upward arrow) occurs on the short-wavelength side of the stop band, as a result of Bragg diffraction in oblique directions. Both effects are quantitatively explained by the escape function model (solid curve). The depth of the stop band $\left(T_{\min }=20 \%\right)$ is the only adjustable parameter in our model.

the direction of the detector. This attenuation in the stop band is determined by the ratio of the Bragg attenuation length $L_{\mathrm{B}}$ and the transport mean free path $l$, which is the average distance that light propagates before losing its initial direction due to scattering by disorder. Most photons in the photonic crystals are emitted by proteins at depths $z$ greater than $l$ and are therefore multiply scattered. The effects of Bragg diffraction can be measured only after the last scattering event, after which the photons emanate from the photonic crystal. The last scattering events most likely occur at $z<l$. Light scattered at $z<L_{\mathrm{B}}$ is hardly Bragg attenuated, while light scattered at $L_{\mathrm{B}}<z<l$ cannot escape in the stopgap direction, so that the depth of the stop band agrees well with our estimate $T_{\min }=1-\left(L_{\mathrm{B}} / l\right)$.

Thus, the depth of the stop band directly reflects the amount of disorder in the sampled crystal position. The enhancement of the escape probability on the blue side of the stop band can be explained in a similar way: this effect is also caused by Bragg diffraction, but in other directions. ${ }^{[26]}$ The measured relative intensity and the calculated escape probability shown in Figure 3 are in excellent agreement. The observation of stop bands in the emission spectrum verifies the successful infiltration of fluorescent proteins into the photonic crystal. Furthermore, the intense emission confirms that the DsRed2 protein cylinders (Figure 1) are still intact after infiltration into the crystal.

\section{Conclusions}

We have shown that the emission properties of fluorescent proteins can be effectively controlled by changing their photonic environment. The color appearance of the fluorescent protein DsRed2 inside photonic crystals changed dramatically with increasing lattice parameter of the crystals, from orange-yellow to red and finally to bright green. The change in color results from the redistribution of light emission around the stop band of the photonic crystal.

The observed colors of the fluorescent proteins are a novel kind of coloring that is caused by a combination of emission colors and interference from the photonic crystals' nanostructure. In comparison, pigment colors are due to selective absorption of light. Structural colors, such as iridescence, are also the result of interference from nanostructures, but they arise in reflection from an external light beam. It is intriguing to speculate on the role of intrinsic nanophotonic structures in the coral backbone in modulating the color of embedded fluorescent proteins and their effect on the color appearance of corals.

We believe that there is a bright future for "biophotonic engineering" whereby fluorescent proteins are used as internal emitters in photonic structures. On the one hand, fluorescent proteins are convenient and efficient probes for fundamental studies of the properties of photonic structures. On the other hand, photobiological processes can be manipulated and enhanced by using the fast-growing toolbox provided by nanophotonics. ${ }^{[8,9]}$ In addition to the inverse opals studied here, versatile one-, two-, and three-dimensional structures can be tailored to accommodate various biological systems. An important future perspective would be the tuning of energy trapping in photosynthesis ${ }^{[27]}$ through the manipulation of the competition between spontaneous emission and Förster energy transfer. ${ }^{[28]}$

\section{Experimental Section}

Titania inverse opals with cubic lattice parameters $a$ between 270 and $620 \mathrm{~nm}$ were made following Ref. [22]. Titania is a biocompatible material-as is illustrated by its everyday use in toothpaste-with a high refractive index and essentially no absorption of visible light. A total of ten sets of crystals with nine different lattice parameters were studied. The uncertainty in the lattice parameter is estimated to range between 10 and $20 \mathrm{~nm}$. The DsRed2 mutant of the reef coral fluorescent protein DsRed was obtained as reported in Ref. [23]. To infiltrate the fluorescent protein into the photonic crystals, the protein solution was first desalted to prevent salt crystallization upon drying and then mixed with ethanol to enhance the capability of the solution to penetrate the photonic crystal. Infiltration of DsRed2 into the inverse opals was achieved by soaking the crystals in the DsRed2 water/ethanol solution. Finally, the crystals were washed with demineralized water to remove fluorescent proteins from the surface of the crystal, and dried in a constant air flow.

To characterize the emission from the fluorescent protein and the reflectivity of the photonic crystals, we used a custom-built setup capable of wide-field reflectivity and fluorescence imaging as well as scanning-stage confocal microscopy. The light sources used were a halogen lamp for reflectivity imaging, a mercury lamp for wide-field fluorescence imaging, and a laser diode emitting at $469 \mathrm{~nm}$ for local excitation when recording emission spectra. The sample was illuminated using a $10 \times$ objective ( 0.3 numerical 
aperture, Olympus), and the emission from the sample was collected by the same objective. Wide-field images were recorded with a color camera (AxioCam HRc, Zeiss). For the emission images, a standard blue filter cube (U-MWB2, Olympus) was used. White balance was optimized for a halogen light temperature of $3200 \mathrm{~K}$, in accordance with the manufacturer's recommendation for fluorescence imaging. We verified the consistency between the color camera image and the coloring visible in the eyepiece of the microscope. Contrast, brightness, and gamma were globally optimized for the whole images, and no digital color-changing filters were applied. To record local emission spectra, the emitted light was imaged by a prism spectrometer onto a cooled CCD camera (Newton EMCCD, Andor). We estimated the area sampled when recording emission spectra to be less than $3 \mu \mathrm{m}$ in diameter. All pictures and spectra were recorded normal to the $h k l=111$ planes of the crystal.

\section{Acknowledgements}

We thank Léon Woldering and Charles Uju for fabricating inverse opals, Raymond Oudeboon for discussion, and Willem Tjerkstra for help. This work is part of the research program of the "Stichting voor Fundamenteel Onderzoek der Materie" (FOM), which is supported by the "Nederlandse Organisatie voor Wetenschappelijk Onderzoek" (NWO). W.L.V. also thanks NWO-Vici and STW/NanoNed. C.B. and V.S. thank the MESA ${ }^{+}$ Institute for Nanotechnology.

[1] M. Chalfie, Y. Tu, G. Euskirchen, W. W. Ward, D. C. Prasher, Science 1994, 263, 802-805.

[2] J. Lippincott-Schwartz, G. H. Patterson, Science 2003, 300, 87-91.

[3] J. Yu, J. Xiao, X. J. Ren, K. Q. Lao, X. S. Xie, Science 2006, 311, 1600-1603.

[4] R. Tsien, Annu. Rev. Biochem. 1998, 67, 509.
[5] M. A. Rizzo, G. H. Springer, B. Granada, D. W. Piston, Nat. Biotechnol. 2004, 22, 445-449.

[6] G. J. Kremers, J. Goedhart, E. B. van Munster, T. W. J. Gadella, Biochemistry 2006, 45, 6570-6580.

[7] M. Matz, A. Fradkov, Y. Labas, A. Savitsky, A. Zaraisky, M. Markelov, S. Lukyanov, Nat. Biotechnol. 1999, 17, 969-973.

[8] E. Yablonovitch, Phys. Rev. Lett. 1987, 58, 2059-2062.

[9] K. J. Vahala, Nature 2003, 424, 839-846.

[10] P. Lodahl, A. F. van Driel, I. S. Nikolaev, A. Irman, K. Overgaag, D. L. Vanmaekelbergh, W. L. Vos, Nature 2004, 430, 654-657.

[11] A. Badolato, K. Hennessy, M. Atature, J. Dreiser, E. Hu, P. M. Petroff, A. Imamoglu, Science 2005, 308, 1158-1161.

[12] P. Vukusic, I. Hooper, Science 2005, 310, 1151.

[13] A. R. Parker, R. C. McPhedran, D. R. McKenzie, L. C. Botten, N. A. P. Nicorovici, Nature 2001, 409, 36-37.

[14] P. Vukusic, J. R. Sambles, Nature 2003, 424, 852-855.

[15] H. Miguez, A. Blanco, F. Meseguer, C. Lopez, H. M. Yates, M. E. Pemble, V. Fornes, A. Mifsud, Phys. Rev. B 1999, 59, 1563-1566.

[16] F. Yang, L. Moss, G. Phillips, Nat. Biotechnol. 1996, 14 $1246-1251$

[17] I. S. Nikolaev, P. Lodahl, W. L. Vos, Phys. Rev. A: At. Mol. Opt. Phys. 2005, 71, 053813.

[18] Y. A. Vlasov, K. Luterova, I. Pelant, B. Honerlage, V. N. Astratov, Appl. Phys. Lett. 1997, 71, 1616-1618.

[19] S. Nishimura, N. Abrams, B. A. Lewis, L. I. Halaoui, T. E. Mallouk, K. D. Benkstein, J. van de Lagemaat, A. J. Frank, J. Am. Chem. Soc. 2003, 125, 6306-6310.

[20] A. Mihi, H. Miguez, J. Phys. Chem. B 2005, 109, 15968-15976.

[21] M. Wall, M. Socolich, R. Ranganathan, Nat. Struct. Biol. 2000, 7, 1133-1138.

[22] J. E. G. J. Wijnhoven, L. Bechger, W. L. Vos, Chem. Mater. 2001, 13, 4486-4499.

[23] C. Blum, A. J. Meixner, V. Subramaniam, J. Am. Chem. Soc. 2006 128, 8664-8670.

[24] W. W. Ward, S. H. Bokman, Biochemistry 1982, 21, 4535-4540.

[25] R. Janes, M. Edge, J. Robinson, N. S. Allen, F. Thompson, J. Mater. Sci. 1998, 33, 3031-3036.

[26] A. F. Koenderink, W. L. Vos, Phys. Rev. Lett. 2003, 91, 213902.

[27] R. van Grondelle, J. P. Dekker, T. Gillbro, V. Sundstrom, Biochim. Biophys. Acta, Bioenerg. 1994, 1187, 1-65.

[28] C. Blum, W. L. Vos, V. Subramaniam, http://arxiv.org/abs/ 0704.3560, 2007.

Received: November 23, 2007 Published online: March 20, 2008 